\title{
MULTIANGULATION POSITION ESTIMATION PERFORMANCE ANALYSIS USING A BARTLETT'S BEAMFORMING METHOD
}

\author{
A. S. Yaro ${ }^{1,}{ }^{*}$, S. Salisu' ${ }^{2}$, A. Umar ${ }^{3}$ and M. J. Musa ${ }^{4}$ \\ 1,2,4 Dept. of Electrical and Computer EngR., AHMAdu Bello University, ZARia, Kaduna STATE, NiGERIA.

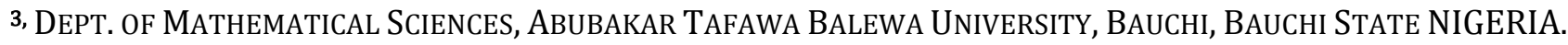 \\ E-mail addresses: 1yaroabdulmalik@yahoo.com, 2 s.salisu@live.com, 3 mmjibrin@yahoo.com, \\ 4 abkamor@gmail.com
}

\begin{abstract}
In this work, a complete multiangulation system was developed and its performance in term of position estimation (PE) was determined. The developed system uses Bartlett's beam forming method to estimate AOA of the signal impinging on the 16-element sensor array in a uniform linear array (ULA) geometry at each ground receiving station (GRS). The AOA measurements are then used as input to a linear angulation algorithm for PE. The PE accuracy of the developed system was determined using Monte Carlo simulation and compared with the directional rotating antenna multiangulation system using a square GRS configuration. Simulation results shows that the developed multiangulation PE error is 50\% lower than that of the directional rotating antenna system. Furthermore, the PE error of the developed system is higher for emitting sources within the system coverage with position bearings within $61^{\circ}$ to $120^{\circ}$ and $240^{\circ}$ to $300^{\circ}$ than other emitting source locations.
\end{abstract}

\section{Keywords: multiangulation system, position estimation, beam forming, Monte Carlo Simulation, position bearing}

\section{INTRODUCTION}

Passive wireless positioning system utilizes an emitting source's electromagnetic (EM) emission detected by the antenna of a receiving station to determine the location of the source. Determining the location of the emitting source is a two-stage process [1].The first stage involves the estimation of a position dependent signal parameter from the received EM radiation such as angle of arrival (AOA), time of arrival (TOA) and receive signal strength (RSS). In the second stage, the position dependent signal parameter estimated from the first stage is used as an input to a position estimation (PE) algorithm such as angulation, fingerprinting and lateration to determine the location of the source of the EM radiation. A multiangulation system is an example of a wireless positioning system that has AOA estimated from the received signal as its position dependent signal parameter and uses the angulation algorithm for PE [1]. Multiangulation system differs from the conventional triangulation system as it uses 4 or more ground receiving stations (GRS) each equipped with a sensor array either in circular or uniform linear array (ULA) geometry for the PE process [2,3]. Another type of wireless positioning system is the Multilateration system that uses time difference of arrival (TDOA) measurements as its position dependent signal parameter with a lateration algorithm for PE [1,4]. It has anadvantage over the multiangulation system as it can easily be used to estimate source location in 3-D.

Various techniques [5-8] have been reported in the literature to perform AOA estimation, which can be grouped as Subspace and classical techniques. The subspace techniques are often referred to as super or high-resolution techniques which exploit the eigenstructure of the input signal matrix for AOA estimation [9-11]. These techniques are useful in case of AOA estimation of multiple sources operating at the same frequency or discrimination of multiple paths from the direct signal [5]. Examples of subspace based AOA estimation techniques [12] include multiple signal classification (MUSIC), estimation of signal parameters via rotational invariance technique (ESPRIT), Pisarenko harmonic decomposition and Root-MUSIC. The classical techniques are based on beamforming and null-steering and require a relatively large number of antenna array elements to achieve high resolution [13]. These techniques include rotating directional antenna, phase interferometry, correlative interferometer, Doppler and pseudo-Doppler, Maximum Likelihood

\footnotetext{
* Corresponding author tel: + 234-806-925-4680
} 
Technique, Bartlett Methodand Capon beamformer [5, 12]. The rotating directional antenna method is the simplest method for AOA estimation among the classical technique but has AOA estimation resolution between $2^{\circ}$ to $5^{\circ}$ [5].The resolution determines how accurate an AOA measurement can be estimated. The lower the value, the higher the accuracy of the AOA estimation method.

After the AOA measurements have been obtained, the second and final stage is the PE process. It involves using the AOA measurement from the first stage with the known GRS positions as input to an angulation algorithm. Due to the non-linear relationship between the AOA measurement and signal source location, several approaches have been used to develop the angulation algorithm which can be grouped as linear and non-linear approaches [1, 14]. The non-linear approach involves the use of linearization algorithms such as the Gauss-Newton algorithm to obtain a linear relationship between the input variable (AOA measurement) and the output (signal source location) $[1,14,15]$. A random source location is then inputted into the linearized equation which is then iteratively refined and the final source location that minimizes the maximum likelihood (ML) cost function is selected as the estimated source location. This approach to angulation algorithm is computationally complex as it involves linearization and an iteration process. It also suffers from convergence issue if the initial source location chosen for the iteration process is far from the actual source location [14]. The linear approach to angulation algorithm was developed to overcome the convergence issue limitation of the non-linear approach. It involves algebraic manipulation of the AOA measurement, GRS coordinates and source location to obtain a linear relationship between the AOA measurement and the source location $[16,17]$.

Most of the published work on AOA-based PE system focused on reducing the PE error of the angulation algorithm and verifying their proposed techniques using one or two source locations [14-16]. Apart from that, the AOA estimation error is assumed to be the same for each AOA estimated at each GRS which is not the case as reported in [18]. In this paper, a complete multiangulation PE system was developed using a beamforming Bartlett method as the AOA estimation technique with a linear approach based angulation PE algorithm. The PE accuracy of the multiangulation system was determined by assuming that the AOA estimation error differs for each AOA of the source estimated at each GRS. Using Monte Carlo simulation, the relationship between the received signal-to-noise ratio (SNR) and beamforming $\mathrm{AOA}$ estimation error was determined for different signal AOA. For a given source location, the received SNR of the signal received at each GRS is determined using the free space path loss model. The received SNR at each GRS is then used to determine the AOA estimation error from which the estimated AOA of the signal at each of the GRS is determined. The estimated AOAs at each of the GRS which contains error are inputted into the angulation algorithm and the PE performance of the multiangulation system was determined using Monte Carlo simulation. Validation of the developed system is carried out by comparison with directional rotating antenna AOA based estimation multiangulation system with equal estimation error for all AOAs which is taken as $2^{0}$ [4].

The rest of the paper is organized as follows. In section 2 , the AOA estimation methodology based on beamforming is described. The multiangulation PE methodology is presented in section 3. This is then followed by simulation parameters, results, and discussion in section 4. Finally, the conclusion is presented in section 5 .

\section{16-ELEMENT ULA BEAMFORMING AOA ESTIMATION METHODOLOGY}

This section of the paper discusses the beamforming AOA estimation technique which was adopted in this paper.

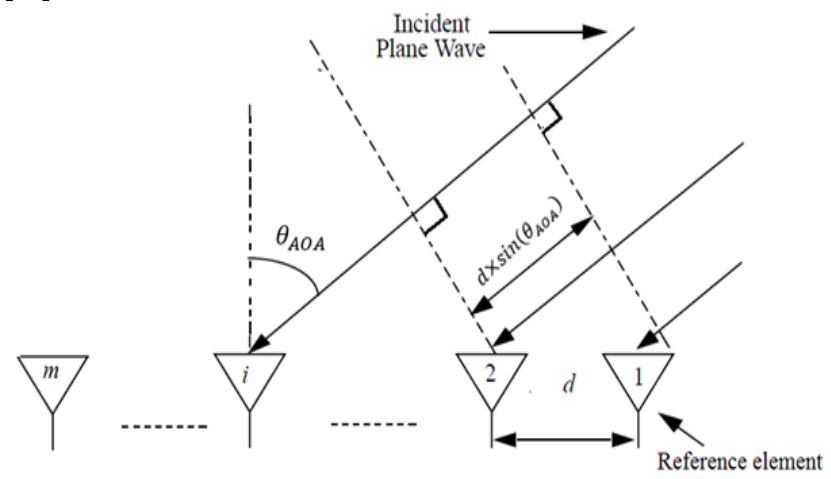

Figure 1: M-element sensor array in ULA geometry.

Given an $m$-element sensor array in a ULA geometry as shown in Figure 1, a radiated narrow band signal defined in Eq. (1) from a source impinges on the sensor array with an angle of arrival, $\theta_{A O A}$.

$$
x(t)=M(t) \exp \left(j 2 \pi f_{c} t\right)
$$

where $x(t)$ is a complex narrow band signal, $M(t)$ is the complex envelope of $x(t)$ and $f_{c}$ is the carrier frequency of the signal.

The signal outputted by the $i$-th element of the sensor array is given as $[8,12]$. For $m=1,2, \ldots 16$,

$$
\hat{x}_{m}(t)=M(t) \exp \left(-j(m-1) \frac{2 n d \sin \left(\theta_{\mathrm{AOA}}\right)}{\lambda}\right)
$$


where $d$ is the distance between the $i$-th and the ( $i-$ 1)-th element which must be less than or equal to half the wavelength $(\lambda)$ of the impinging signal.

Let the response of the $i$-th element in the sensor array to the impinging signal be $[8,12]$

$$
a_{i}(\theta)=\exp \left(-\mathrm{j}(\mathrm{j}-1) \frac{2 \pi \mathrm{d} \sin (\theta)}{\lambda}\right)
$$

and the distance between the $i-t h$ and the $(i+1)-t h$ ULA antenna element be

$$
d=\frac{\lambda}{2}
$$

The total response of the $M=16$ number array elements which is known as the steering vector can be obtained in vector form as $[8,12]$

$$
\begin{gathered}
a_{\mathrm{ULA}}(\theta)=1, \exp (-j \pi \sin (\theta)), \exp (-j 2 \pi \sin (\theta)), \ldots \\
\exp (-j 15 \pi \sin (\theta))
\end{gathered}
$$

AOA estimation involves steering the sensor array from $\theta=0^{0}$ to $180^{\circ}$ and the power at each angle is calculated [12]. The steering bearing which results in the maximum power corresponds to the AOA of the impinging signal on the sensor array. The mean output power of the sensor array elements is given as

$$
P(\theta)=w(\theta){ }^{H} R w(\theta)
$$

where(. $)^{H}$ denotes conjugate transposition of matrix and $\mathbf{R}$ is the covariance matrix defined as

$$
\mathrm{R}=E\left[\mathrm{x}(t) \mathrm{x}(t)^{H}\right]
$$

and $\mathrm{w}(\theta)$ is the weighting vector which can be calculated using several algorithms some of which are MUSIC algorithm, Bartlett's algorithm and ESPRIT algorithm [12]. In this paper, Bartlett's algorithm also known as conventional beamforming is used to estimate the weighing vector due to computational ease and robustness to input noise [12]. The Bartlett's beamforming algorithm weighing vector is obtained as [12].

$$
w(\theta)=\frac{a_{\mathrm{ULA}}(\theta)}{\sqrt{a_{\mathrm{ULA}}(\theta)^{H} a_{\mathrm{ULA}}(\theta)}}
$$

In practice, the received signal impinging on the array are affected by some propagation impairments thus resulting in error in the AOA estimation. The major contributor to AOA measurement error in this research is assumed to be thermal noise which is modeled as additive white Gaussian noise (AWGN) and is quantified by the received SNR. Thus, the estimated $\mathrm{AOA}$ is given as

$$
\hat{\theta}_{A O A}=\theta_{A O A}+N\left(O, \theta_{\text {std }}\right)
$$

where $\theta_{A O A}$ is the actual AOA and $\theta_{\text {std }}$ is the AOA error standard deviation which is a function of the received SNR at the sensor array. For $N$ number of GRSs, each GRS will have a different AOA error standard deviation which is a function of the SNR of received signal impinging on the sensor array as shown in Eq. (10)

$$
\theta_{\text {st }, i}=f\left(S N R_{i}\right) \text { for } i=1,2, \cdots, N
$$

For SNR calculation using the free space path loss model, the GRS antenna gain needs to be calculated. The gain of a $M$-element sensor array in $\mathrm{dBi}$ is obtained as:

$$
G_{r}=10 \times \log _{10}(M)
$$

The estimated AOAs in this section are used to determine the location of the source in the next section.

\section{MULTIANGULATION POSITION ESTIMATION METHODOLOGY}

This section describes the methodology for estimating the location of an emitting source using the AOA estimated in Section 2. $N=4$, numbers of GRSs are used with each GRS consisting of a 16-element sensor array in a ULA geometry for the AOA estimation.

Let the coordinate of the $i$-th GRS be $\left(x_{i}, y_{i}\right)$ and the coordinate of a stationary emitting source be $(x, y)$. The line of bearing (LOB) between the stationary emitter and the $i$-th GRS is in the form of the equation of a straight line as defined in Eq. (12).

$$
y=m_{i} x-c_{i}
$$

where $m_{i}=\tan \left(\theta_{i}\right)$ is the gradient, $c_{i}=y_{i}-\tan \left(\theta_{i}\right)$ is the $y$-axis intercept and $\theta_{i}$ is the AOA of the emitter at the i-th GRS.

Since the AOA measurement contains error as defined in Eq. (9),the LOB represented by Eq. (12) can be rewritten as

$$
y=\widehat{m}_{i} x-\hat{C}_{i}
$$

where $\hat{m}_{i}=\tan \left(\hat{\theta}_{i}\right)$ is the gradient and $\hat{c}_{i}=y_{i}-$ $\tan \left(\hat{\theta}_{i}\right)$ is the $y$-axis intercept.

With 4 number of GRSs, 4 LOB equations are obtained which are:

$$
\left\{\begin{array}{l}
y=\tan \left(\hat{\theta}_{1}\right) x-y_{1}+\tan \left(\hat{\theta}_{1}\right) \\
y=\tan \left(\hat{\theta}_{2}\right) x-y_{2}+\tan \left(\hat{\theta}_{2}\right) \\
y=\tan \left(\hat{\theta}_{3}\right) x-y_{3}+\tan \left(\hat{\theta}_{3}\right) \\
y=\tan \left(\hat{\theta}_{4}\right) x-y_{4}+\tan \left(\hat{\theta}_{4}\right)
\end{array}\right.
$$

where $\hat{\theta}_{1}, \hat{\theta}_{2}, \hat{\theta}_{3}$ and $\hat{\theta}_{4}$ are the estimated AOA of the emitting source from GRS labelled 1, GRS labelled 2, GRS labelled 3, and GRS labelled 4 respectively. The unknown from Eq. (14) are the emitting source coordinates $x$ and $y$.

Eq. (12) can be presented in matrix form as

$$
\left[\begin{array}{ll}
-\tan \left(\hat{\theta}_{1}\right) & 1 \\
-\tan \left(\hat{\theta}_{2}\right) & 1 \\
-\tan \left(\hat{\theta}_{3}\right) & 1 \\
-\tan \left(\hat{\theta}_{4}\right) & 1
\end{array}\right]\left[\begin{array}{l}
x \\
y
\end{array}\right]=\left[\begin{array}{ll}
\tan \left(\hat{\theta}_{1}\right) & \mathrm{y}_{1} \\
\tan \left(\hat{\theta}_{2}\right) & \mathrm{y}_{2} \\
\tan \left(\hat{\theta}_{3}\right) & \mathrm{y}_{3} \\
\tan \left(\hat{\theta}_{4}\right) & \mathrm{y}_{4}
\end{array}\right] \text { or } \mathrm{Ax}=\mathrm{b}
$$

Eq. (15) represents the multiangulation PE matrix model for 4 number GRSs. It involves solving for the source location $(x, y)$ given the AOA measurements and the GRS coordinates. Eq. (15) is an overdetermined 
least square (LS) problem which can be solved using the pseudo-inverse LS approach defined by Eq. (16).

$$
\hat{\mathrm{x}}=\left(\mathrm{A}^{\mathrm{T}} \mathrm{A}\right)^{2} \mathrm{Ab}
$$

Since the AOA measurements contain error defined by Eq. (8), the location of the source obtained using Eq. (14) will contain error. The effect of the AOA measurement error on the $\mathrm{PE}$ of the multiangulation will be determined in the next section.

\section{SIMULATION PARAMETERS, RESULTS AND DISCUSSION}

In this section, the parameters for the simulation are first presented following by the relationship between the beamforming AOA estimation error with the received SNR. Finally, the effect of the AOA measurement error on the PE accuracy of the multiangulation is determined.

\subsection{Simulation Parameter}

The PE root mean square error (RMSE) and AOA are the performance measures used to determine the PE accuracy of the multiangulation system and the beamforming AOA estimation method. Mathematically, the PE and AOA RMSE respectively are defined as

$$
\begin{gathered}
P E_{\text {rmse }}=\sqrt{\frac{\sum_{i=1}^{N}\left[\left(x-x_{i}\right)^{2}+\left(x-y_{i}\right)^{2}\right]}{N}} \\
A O A_{\text {rmse }}=\sqrt{\frac{\sum_{i=1}^{N}\left(\theta-\theta_{i}\right)^{2}}{N}}
\end{gathered}
$$

where $(x, y)$ and $\theta$ respectively are the known source location and AOA of the impinging signal on the sensor array, $\left(x_{i}, y_{i}\right)$ and $\theta_{i}$ are respectively the estimated source location and AOA at the $i$-th Monte Carlo realization and $N$ is the number of Monte Carlo simulation realization.

The distribution of all the 4 GRSs in a square configuration can be seen in Figure 2.

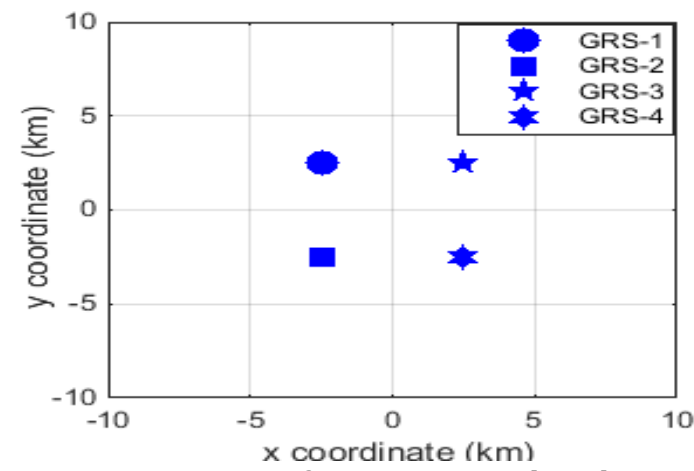

Figure 2: Square configuration GRS distribution [5 km separation].

The Source locations for determining the PE accuracy of the multiangulation system are presented in cylindrical coordinate $\operatorname{system}(R, \theta) . R(\mathrm{~km})$ is the source horizontal range and $\theta\left(^{\circ}\right)$ is the source bearing all taken from the center of the GRS configuration in Figure 2. Conversion from cylindrical to rectangular coordinate system is possible using Eq. (16)

$$
\begin{aligned}
& x=R \times \sin (\theta) \\
& y=R \times \cos (\theta)
\end{aligned}
$$

The transmitter and receiver parameters for simulation are presented in Table 1 [18-20].

Table 1: Simulation parameter

\begin{tabular}{lll}
\hline No. & Parameters & Value \\
\hline 1 & Transmit power & $30 \mathrm{dBm}$ \\
2 & Carrier frequency & $928 \mathrm{~Hz}$ \\
3 & GRS receiver sensitivity & $-90 \mathrm{dBm}$ \\
4 & GRS antenna gain & $12 \mathrm{dBi}$ \\
5 & Transmitter antenna gain & $6 \mathrm{dBi}$ \\
\hline
\end{tabular}

\subsection{Emitter Position Versus Received SNR}

It is assumed that there is line of sight (LOS) between the emitting source and all the GRS. Thus, the free space path loss model is used. The SNR of the signal at each GRS is different due to the geometrical relationship between the source location and the GRS configuration. Figure 3 show the SNR of the signal received at each GRS based on Figure 1 for source horizontal range of $0 \mathrm{~km}$ to $30 \mathrm{~km}$ and bearing of $0^{0}$ to $359^{\circ}$. At emitter position $\left(R=20 \mathrm{~km}, \theta=45^{\circ}\right)$ which corresponds to $(x=14.14 \mathrm{~km}, y=14.14 \mathrm{~km})$ in rectangular coordinate system, the received SNR at GRS 1, GRS 2, GRS 3, GRS 4 are $20 \mathrm{~dB}, 18 \mathrm{~dB}, 21 \mathrm{~dB}$ and 20 $\mathrm{dB}$ respectively. This means that there is slight difference in the received SNR of the signal at each GRS. The differences in the received SNR will result to different RMSE of the AOA estimated at each GRS.

The received SNR of the signal impinging on the sensor array at each GRS is used to determine the RMSE of the AOA measurement. The relationship between the received SNR and AOA RMSE is determined in the next section for some selected AOA.

\subsection{Beamforming AOA RMSE Versus Received Signal SNR}

100 realization Monte Carlo simulation was carried out to determine the relation between the received SNR impinging on the sensor array and the AOA RMSE of the beamforming Bartlett method. The sensor array used in this work has $m=16$ number of elements. By varying the received SNR from $1 \mathrm{~dB}$ to $35 \mathrm{~dB}$, the corresponding RMSE is determined for AOA of $30^{\circ}, 45^{\circ}$ and $60^{\circ}$. Figure 4 presents the relationship between the AOA RMSE versus the received SNR for the selected AOAs. For all the selected AOAs, the AOA RMSE decreases as the SNR increases from $1 \mathrm{~dB}$ to $25 \mathrm{~dB}$. 


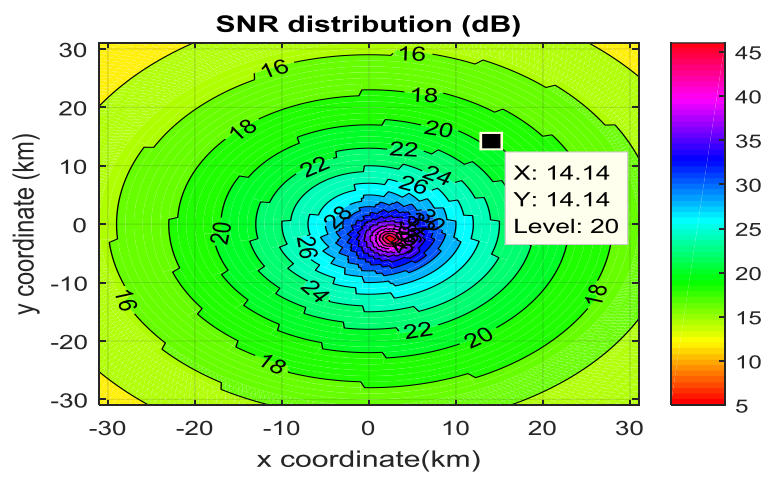

(a) Received SNR at GRS 1

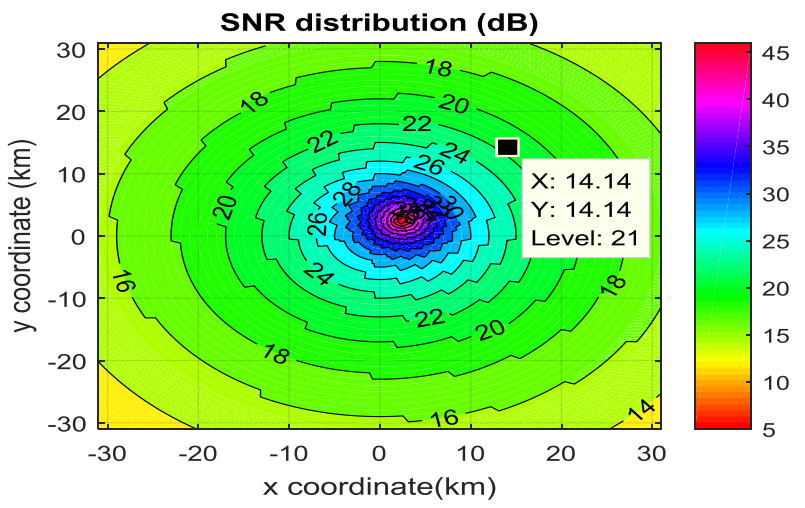

(c) Received SNR at GRS 3

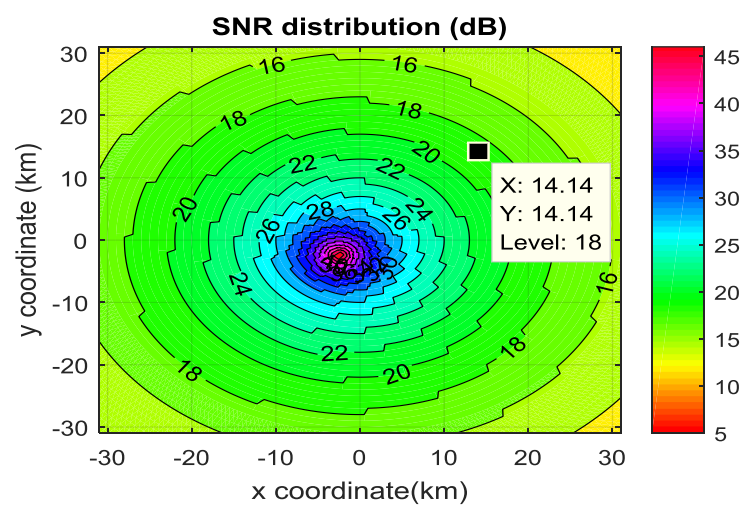

(b) Received SNR at GRS 2

SNR distribution (dB)

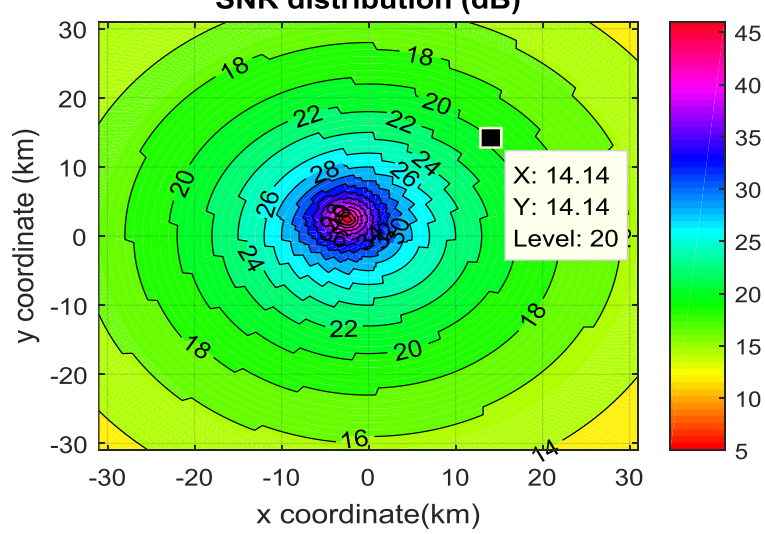

(d) Received SNR at GRS 4

Figure 3. SNR of the received signal at each GRS [5 km separation]

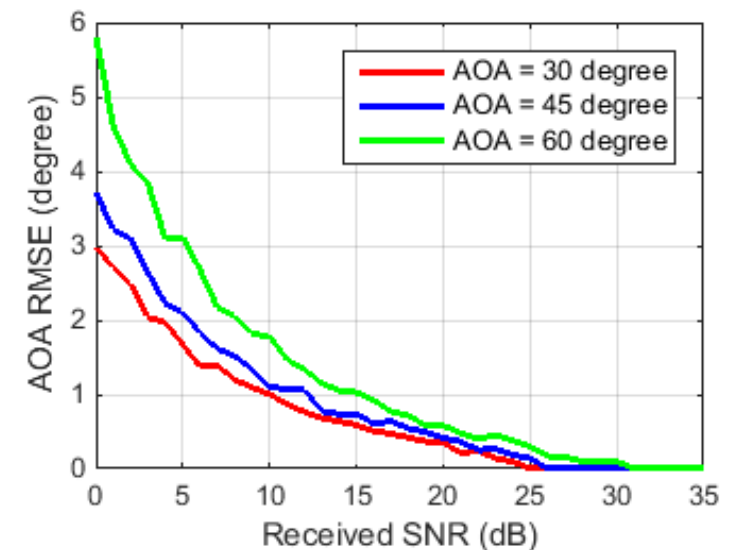

Figure 4: AOA RMSE versus received SNR for some selected AOA measurement.

The AOA RMSE at SNR of $15 \mathrm{~dB}$ for AOA of $30^{\circ}, 45^{\circ}$ and $60^{\circ}$ are $0.59^{\circ}, 0.75^{\circ}$ and $1.10^{\circ}$ respectively. This means that at the same SNR value, the AOA RMSE for $30^{\circ}, 45^{\circ}$ and $60^{\circ}$ are different. Thus, the AOA RMSE of the beamforming AOA estimation depends on the AOA and the received SNR. Due to the geometrical configuration of the GRSs, the AOA of the signal transmitted by an emitter is different at each of the GRSs. For instance, based on the GRS configuration in Figure 2, an emitter at position $\left(R=5 \mathrm{~km}, \theta=30^{\circ}\right)$ will have AOA of $90^{\circ}$, $54^{\circ}, 90^{\circ}$ and $20^{\circ}$ at GRS 1, GRS 2, GRS 3 and GRS 4 respectively. At each GRS, thereceived SNR is first estimated, which is then used to determine the RMSE of the AOA of the signal impinging the sensor array at that GRS. Using the AOA RMSE, the PE accuracy of the multiangulation system is determined and is presented in the next section.

\subsection{Multiangulation System Position Estimation Accuracy}

The effect of AOA RMSE estimated in Section 4.3 at each GRSs on the PE accuracy of the multiangulation system is presented in this section. Its PE accuracy will be compared with a multiangulation system that uses the directional rotating antenna with a resolution of $2^{0}$ for all AOA estimations. 100 realization Monte Carlo simulation was carried to obtain the PE RMSE for both multiangulation systems for radiating source locations within the range of $0 \mathrm{~km}$ to $30 \mathrm{~km}$ and bearings of $0^{0}$ to 
$359^{0}$. Figures 5 and 6 respectively shows the horizontal RMSE for the developed multiangulation system and that of the directional rotating antenna AOA based estimation method using the GRS configuration shown in Figure 2. Simulation result comparison shows that the PE error depends on the source location. It increases with increase in the source range from $0 \mathrm{~km}$ to $30 \mathrm{~km}$. Table 2 shows PE RMSE comparison between the two multiangulation systems for some selected source location. The PE error for the selected source location indicated higher PE error for the rotating directing antenna compared to the developed multiangulation system. This is due to the fact that each GRS estimates difference AOA for the emitting source at a particular location and the estimation error for these AOAs are different. Some AOA will have a higher estimation error while some will have a low estimation error. The AOA with the lower estimation error contributes significantly to decreasing the PE error of the entire system which is not the case of assuming equal AOA estimation error as shown in Figure 6.

\section{CONCLUSION}

In this work, a complete multiangulation system was developed and the PE performance of the system was

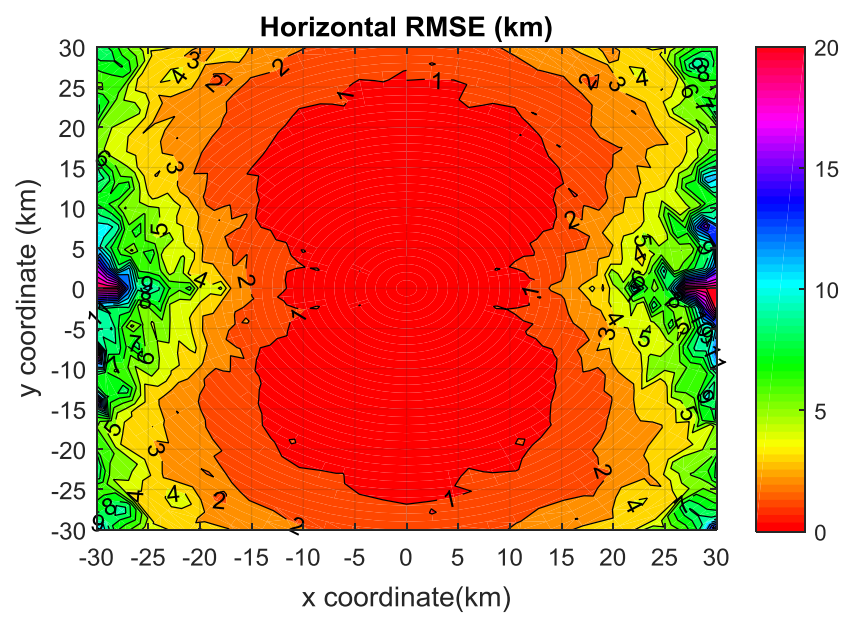

Figure 5: Horizontal PE RMSE for the developed multiangulation system with beamforming Bartlett's AOA estimation method. carried out using 4 GRSs each equipped with a 16element sensor array in a ULA geometry. Using Bartlett's beamforming method, AOA of the impinging signal at each GRS was obtained which is then used with a linear angulation algorithm to determine the location of the signal source. The PE performance of the system was carried out for emitting sources located within $30 \mathrm{~km}$ coverage radius of the system with the GRS in a square configuration and compare with the directional rotating antenna based multiangulation system. The directional rotating antenna system is assumed to have equal estimation error of about $2^{2}$ for all AOA estimation. Simulation results comparison shows that the developed multiangulation system based on the GRS configuration has lower PE error than the rotating directional antenna system. The PE accuracy of the multiangulation system very much depends on the AOA estimation technique used. Subspace methods have high AOA estimation accuracy due to their high resolution thus, if used in the multiangulation system for AOA estimation process will result in lower PE error.

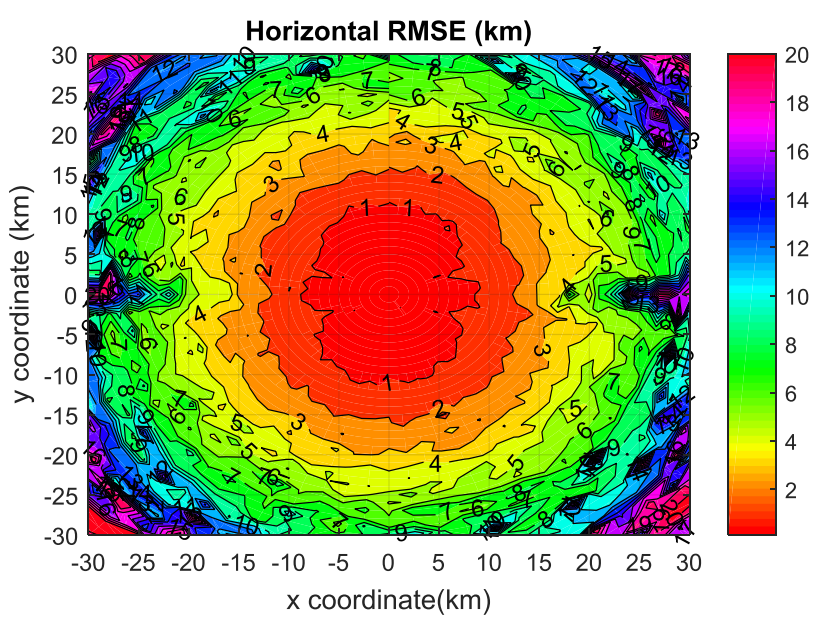

Figure 6: Horizontal PE RMSE for the directional rotating antenna multiangulation system with $A O A$ estimation error of $2^{\circ}$

Table 2: PE error for some selection source locations

\begin{tabular}{|c|c|c|c|c|c|}
\hline \multirow[b]{2}{*}{ No. } & \multirow[b]{2}{*}{ Range (km) } & \multirow[b]{2}{*}{ Bearing $\left(^{\circ}\right)$} & \multicolumn{2}{|c|}{ Horizontal RMSE $(\mathrm{km})$} & \multirow[b]{2}{*}{ PE difference $(\mathrm{km})$} \\
\hline & & & $\begin{array}{c}\text { Develop multiangulation } \\
\text { System }\end{array}$ & $\begin{array}{c}\text { Directional rotating antenna } \\
\text { multiangulation system }\end{array}$ & \\
\hline 1 & \multirow{2}{*}{10} & 30 & 0.12 & 0.91 & 0.79 \\
\hline 2 & & 80 & 0.51 & 1.38 & 0.87 \\
\hline 3 & \multirow{2}{*}{20} & 30 & 0.66 & 3.56 & 2.9 \\
\hline 4 & & 80 & 2.93 & 4.06 & 1.13 \\
\hline 5 & \multirow{2}{*}{30} & 270 & 20 & 20 & 0 \\
\hline 6 & & 330 & 1.80 & 8.35 & 6.55 \\
\hline
\end{tabular}




\section{REFERENCES}

[1] Dardari, D., Falletti, E. and Luise, M. Satellite and terrestrial radio positioning techniques: a signal processing perspective. Academic Press, 2011.

[2] Campos, R., RF positioning, 1st ed. Boston: Artech House, 2016.

[3] Yaro, A. S. and Zuri, S. A., "Mathematical Model of Position Estimation Error for a Multiangulation System," in the 6th International Graduate Conference on Engineering, Science \& Humanity: Empowering innovation and Entrepreneurship for sustainable Development, Universiti Teknologi Malaysia (UTM), Johor Bahru, Malaysia, Universiti Teknologi Malaysia (UTM), pp. 134-136.

[4] Sha'ameri, A. Z., Shehu, Y. A., and Asuti, W. "Performance analysis of a minimum configuration multilateration system for airborne emitter position estimation," Defence $S$ and $T$ Technical Bulletin, vol. 8, Number 1, 2015, pp. 2741.

[5] ITUR-R. Handbook on spectrum monitoring. ITU Radio communication Bureau, Geneva, 2010.

[6] David, M., Lara, F. B., Vargas, C., and EnriquezCaldera, R., Position location techniques and applications. Academic Press, 2009.

[7] Friedlander, B. and Tuncer, T., Classical and modern direction-of-arrival estimation, 1st ed. New York: Academic Press, 2009.

[8] Poisel, R., Electronic warfare target location methods. Boston, M. A: Artech House Publishers, 2005.

[9] Chuang, S., Wu, W. and Liu, Y., "High-Resolution AoA Estimation for Hybrid Antenna Arrays", IEEE Transactions on Antennas and Propagation, vol. 63, no. 7, pp. 2955-2968, 2015.

[10] Mohanna, M., Rabeh, M., Zieur, E. and Hekala, S., "Optimization of MUSIC algorithm for angle of arrival estimation in wireless communications", NRIAG Journal of Astronomy and Geophysics, vol. 2, no. 1, pp. 116-124, 2013.

[11] Wang, Q., Wang, L., An, K., Shou, Z. and Zhang, H., "DOA Estimation of Smart Antenna Signal Based on MUSIC Algorithm", Journal of Networks, vol. 9, no. $5,2014$.
[12] Khmou, Y., Safi, S. andFrikel, M., "Comparative study between several direction of arrival estimation methods", Journal of Telecommunications and Information Technology, vol. 1, no. 2014, pp. 41-48, 2017.

[13] Singh, H. and Jha, R., "Trends in Adaptive Array Processing", International Journal of Antennas and Propagation, vol. 2012, pp. 1-20, 2012.

[14] Wang, Y. and Ho, K., "An Asymptotically Efficient Estimator in Closed-Form for 3-D AOA Localization Using a Sensor Network", IEEE Transactions on Wireless Communications, vol. 14, no. 12, pp. 6524-6535, 2015.

[15] Doğançay, K. and Hmam, H., "Optimal angular sensor separation for AOA localization", Signal Processing, vol. 88, no. 5, pp. 1248-1260, 2008.

[16] He, Y., Behnad, A. and Wang, X., "Accuracy Analysis of the Two-Reference-Node Angle-ofArrival Localization System", IEEE Wireless Communications Letters, vol. 4, no. 3, pp. 329-332, 2015.

[17] Kułakowsk, P.i, Vales-Alonso, J., Egea-López, E., Ludwin, W. and García-Haro, J., "Angle-of-arrival localization based on antenna arrays for wireless sensor networks", Computers \& Electrical Engineering, vol. 36, no. 6, pp. 1181-1186, 2010.

[18] Finne, M., Methods for direction-finding of directsequence spread-spectrum signals, 1st ed. Diane Pub Co, 1996.

[19] SAAB, "Multilateration System High Performance Positioning on the Ground and in the Air," [Online]. Available:http://saab.com/security/airtraffic-management/air-trafficmanagement/Wide-Area-multilateration/. Accessed: Nov. 20, 2016.

[20] "Electronic Code of Federal Regulations", 2017. [Online]. Available: https://www.ecfr.gov/cgibin/text-dx?SID=eed706a2c49fd927110 6c3228b0615f $3 \& \mathrm{mc}=$ true \& $\quad$ node $=p t 47.1$. 15\&rgn=div5\#se47.1.15_1111. [Accessed: 02Apr- 2017].

[21] ADS-B Subsystem, SELEX Sistemi Integrati Standard E5010015201SDD, 2014. 